\title{
Experimental detection of quantum channel capacities
}

\author{
Álvaro Cuevas, ${ }^{1}$ Massimiliano Proietti, ${ }^{2,1}$ Mario Arnolfo Ciampini, ${ }^{1}$ Stefano \\ Duranti, ${ }^{1,3}$ Paolo Mataloni, ${ }^{1}$ Massimiliano F. Sacchi, ${ }^{4}$ and Chiara Macchiavello ${ }^{5}$ \\ ${ }^{1}$ :Quantum Optics Group, Dipartimento di Fisica, Università di Roma La Sapienza, \\ Piazzale Aldo Moro 5, I-00185 Roma, Italy. \\ ${ }^{2}$ :Edinburgh Mostly Quantum Lab, School of Engineering and Physical Sciences, \\ Heriot-Watt University, David Brewster Building, EH14 4 AS Edinburgh, United Kingdom \\ ${ }^{3}$ :Dipartimento di Fisica e Geologia, Università degli Studi di Perugia, \\ Via Pascoli snc, I-06123 Perugia, Italy. \\ ${ }^{4}$ :Istituto di Fotonica e Nanotecnologie - Consiglio Nazionale delle Ricerche, \\ Piazza Leonardo da Vinci 32, I-20133, Milano, Italy. \\ ${ }^{5}$ :Dipartimento di Fisica, Università di Pavia, and INFN - Sezione di Pavia \\ Via A. Bassi 6, I-27100 Pavia, Italy
}

(Dated: May 23, 2022)

\begin{abstract}
We present an efficient experimental procedure that certifies non vanishing quantum capacities for qubit noisy channels. Our method is based on the use of a fixed bipartite entangled state, where the system qubit is sent to the channel input. A particular set of local measurements is performed at the channel output and the ancilla qubit mode, obtaining lower bounds to the quantum capacities for any unknown channel with no need of a quantum process tomography. The entangled qubits have a Bell state configuration and are encoded in photon polarization. The lower bounds are found by estimating the Shannon and von Neumann entropies at the output using an optimized basis, whose statistics is obtained by measuring only the three observables $\sigma_{x} \otimes \sigma_{x}, \sigma_{y} \otimes \sigma_{y}$ and $\sigma_{z} \otimes \sigma_{z}$.
\end{abstract}

Introduction: Any communication channel is unavoidably affected by noise that limits its ability to transmit information, quantified in terms of channel capacity. When the use of the channel aims to convey quantum information, its efficiency is evaluated in terms of the quantum capacity, which is the maximum number of qubits that can be reliably transmitted per channel use [14, and represents a central quantitative notion in quantum communications. In general the computation of the quantum capacity is a hard task since it requires a regularisation procedure over an infinite number of channel uses, and it is therefore by itself not directly accessible experimentally. Its analytical value is known mainly for some channels that have the property of degradability [5] 7], since regularisation is not needed in this case.

In this Letter we address the issue of experimental detection of Quantum Channel Capacities. For a generic unknown channel the quantum capacity can be in principle estimated via quantum process tomography [8 18, which provides a complete reconstruction of the channel, and therefore leads to an evaluation of all its communication properties. This, however, is a demanding procedure in terms of the number of required different measurement settings, since it scales as $d^{4}$ for a finite $d$-dimensional quantum system. Moreover, being an indirect method, it also has the drawback of involving larger errors due to error propagation.

Here, we are not interested in reconstructing the complete form of the noise affecting the channel, but only in detecting its quantum capacity, which is a very specific feature for which we developed a novel and less demanding procedure in terms of resources (measurements) involved. This is pursued in the same spirit as it is done, for example, in entanglement detection for composite sys- tems [19], in parameter estimation procedures [20], and in the detection of specific properties of quantum channels, such as being entanglement-breaking 21] or nonMarkovian 22.

In this Letter we report the first experiment where a lower bound to the quantum channel capacity is directly accessed by means of a number of local measurements that scales as $d^{2}$, hence more favourably w.r.t. process tomography that scales as $d^{4}$. The experiment is based on a recently proposed theoretical method 23 that can be applied to generally unknown noisy channels in arbitrary finite dimension, and has been proved to be very efficient for many examples of qubit channels [24].

The method is suited for any kind of physical system available for quantum communication, and the experiment we present here is based on a quantum optical implementation for various forms of noisy single-qubit channels.

Lower Bound on Quantum Channel Capacity: The quantum capacity $Q$ of a noisy channel $\mathcal{E}$, measured in qubits per channel use, is defined as [1,4]

$$
Q=\lim _{N \rightarrow \infty} \frac{Q_{N}}{N},
$$

where $N$ is the number of channel uses, $Q_{N}=$ $\max _{\rho} I_{c}\left(\rho, \mathcal{E}_{N}\right)$, with $\mathcal{E}_{N}=\mathcal{E}^{\otimes N}$, and $I_{c}\left(\rho, \mathcal{E}_{N}\right)$ denotes the coherent information [25]

$$
I_{c}\left(\rho, \mathcal{E}_{N}\right)=S\left[\mathcal{E}_{N}(\rho)\right]-S_{e}\left(\rho, \mathcal{E}_{N}\right) .
$$

In the above equation $S(\rho)=-\operatorname{Tr}\left[\rho \log _{2} \rho\right]$ is the von Neumann entropy and $S_{e}(\rho, \mathcal{E})$ represents the entropy exchange [26], i.e. $S_{e}(\rho, \mathcal{E})=S\left[\left(\mathcal{I}_{R} \otimes \mathcal{E}\right)\left(\left|\Psi_{\rho}\right\rangle\left\langle\Psi_{\rho}\right|\right)\right]$, where 
$\left|\Psi_{\rho}\right\rangle$ denotes any purification of $\rho$ by means of an ancilla reference quantum system $A$, namely $\rho=\operatorname{Tr}_{A}\left[\left|\Psi_{\rho}\right\rangle\left\langle\Psi_{\rho}\right|\right]$.

The following chain of bounds holds

$$
Q \geq Q_{1} \geq I_{c}\left(\rho, \mathcal{E}_{1}\right) \geq Q_{D E T},
$$

where the first two inequalities come directly from the above definitions, while the last one was proved in [23], with

$$
Q_{D E T}=S[\mathcal{E}(\rho)]-H(\vec{p}) .
$$

Here $\mathcal{E}(\rho)$ is the output state for a single use of the channel and $H(\vec{p})$ denotes the Shannon entropy for the vector of the probabilities $\left\{p_{i}\right\}$ corresponding to a measurement on orthogonal projectors $\left\{\left|\Phi_{i}\right\rangle\right\}$ in the tensor product of the ancilla and the system Hilbert spaces:

$$
p_{i}=\operatorname{Tr}\left[\left(\mathcal{I}_{A} \otimes \mathcal{E}\right)\left(\left|\Psi_{\rho}\right\rangle\left\langle\Psi_{\rho}\right|\right)\left|\Phi_{i}\right\rangle\left\langle\Phi_{i}\right|\right] .
$$

The procedure to detect the lower bound $Q_{D E T}$ is the following: 1) prepare a bipartite pure state $\left|\Psi_{\rho}\right\rangle ; 2$ ) send it through the channel $\mathcal{I}_{A} \otimes \mathcal{E}$, where the unknown channel $\mathcal{E}$ acts on one of the two subsystems; 3) measure suitable local observables on the joint output state in order to estimate $S[\mathcal{E}(\rho)]$ and $\vec{p}$, and to compute $Q_{D E T}$. After the measurements have been performed, the detected bound $Q_{D E T}$ can then be optimized over all probability distributions that can be obtained from the used measurement settings. This last step is achieved by performing an ordinary classical processing of the measurement outcomes. We now specify our scenario to qubit channels $(d=2)$,

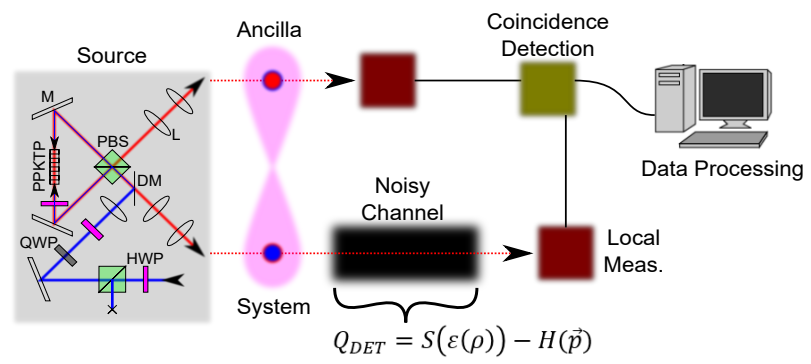

FIG. 1. Experimental Setup. A Sagnac interferometric source of polarization entangled photons sends the S-qubit through the noisy channel, while the A-qubit remains untouched. Both qubits are measured in a joint photon counter system. Here PPKTP is a periodically poled potassium titanyl phosphate non-linear crystal, PBS a polarizing beam splitter, M a mirror, L a converging lens, HWP a half-wave plate, QWP a quarter-wave plate, and DM a dichroic mirror.

where the protocol only requires $d^{2}-1=3$ observables, in our case $\sigma_{x} \otimes \sigma_{x}, \sigma_{y} \otimes \sigma_{y}$, and $\sigma_{z} \otimes \sigma_{z}$ on both the ancilla and system qubit. We also consider a maximally entangled input state $\left|\Phi^{+}\right\rangle=1 / \sqrt{2}(|00\rangle+|11\rangle)$. The schematic representation of the procedure is shown in Fig. 11. The above observables allow one to measure $\left\{\sigma_{i}\right\}$ on the system alone by ignoring the statistics of the measurement results on the ancilla. Since this set is tomographically complete, the system output state $\mathcal{E}(\rho)$ can be reconstructed, and therefore the term $S[\mathcal{E}(\rho)]$ in Eq. (4) can be exactly estimated. Moreover, the measurement settings $\left\{\sigma_{x} \otimes \sigma_{x}, \sigma_{y} \otimes \sigma_{y}, \sigma_{z} \otimes \sigma_{z}\right\}$ allow us to estimate the vector $\vec{p}$ pertaining to the projectors onto the following inequivalent bases 23

$$
\begin{aligned}
B_{1}= & \left\{\left|B_{1,1}\right\rangle,\left|B_{1,2}\right\rangle,\left|B_{1,3}\right\rangle,\left|B_{1,4}\right\rangle\right\} \\
= & \left\{a\left|\Phi^{+}\right\rangle+b\left|\Phi^{-}\right\rangle,-b\left|\Phi^{+}\right\rangle+a\left|\Phi^{-}\right\rangle,\right. \\
& \left.c\left|\Psi^{+}\right\rangle+d\left|\Psi^{-}\right\rangle,-d\left|\Psi^{+}\right\rangle+c\left|\Psi^{-}\right\rangle\right\} ; \\
B_{2}= & \left\{\left|B_{2,1}\right\rangle,\left|B_{2,2}\right\rangle,\left|B_{2,3}\right\rangle,\left|B_{2,4}\right\rangle\right\}, \\
= & \left\{a\left|\Phi^{+}\right\rangle+b\left|\Psi^{+}\right\rangle,-b\left|\Phi^{+}\right\rangle+a\left|\Psi^{+}\right\rangle,\right. \\
& \left.c\left|\Phi^{-}\right\rangle+d\left|\Psi^{-}\right\rangle,-d\left|\Phi^{-}\right\rangle+c\left|\Psi^{-}\right\rangle\right\} ; \\
B_{3}= & \left\{\left|B_{3,1}\right\rangle,\left|B_{3,2}\right\rangle,\left|B_{3,3}\right\rangle,\left|B_{3,4}\right\rangle\right\} \\
= & \left\{a\left|\Phi^{+}\right\rangle+i b\left|\Psi^{-}\right\rangle, i b\left|\Phi^{+}\right\rangle+a\left|\Psi^{-}\right\rangle,\right. \\
& \left.c\left|\Phi^{-}\right\rangle+i d\left|\Psi^{+}\right\rangle, i d\left|\Phi^{-}\right\rangle+c\left|\Psi^{+}\right\rangle\right\} ;
\end{aligned}
$$

where $\left|\Phi^{ \pm}\right\rangle=1 / \sqrt{2}(|00\rangle \pm|11\rangle)$ and $\left|\Psi^{ \pm}\right\rangle=1 / \sqrt{2}(|01\rangle \pm$ $|10\rangle)$ denote the Bell states, and $a, b, c, d$ are real numbers, such that $a^{2}+b^{2}=c^{2}+d^{2}=1$.

The evaluation of the Shannon entropy $H(\vec{p})$ in the $B_{i}$ basis is obtained from its definition

$$
H\left(\vec{p}_{i}\right)=-\sum_{j} p_{i, j} \log _{2} p_{i, j},
$$

where $\overrightarrow{p_{i}}=\left\{p_{i, j}\right\}$ is the probability vector associated to $\left(\mathcal{I}_{A} \otimes \mathcal{E}\right)\left(\left|\Phi^{+}\right\rangle\left\langle\Phi^{+}\right|\right)$, described in Eq. (5).

The probability vectors can be obtained by measuring the expression $p_{i, j}=\left\langle\Pi_{i, j}\right\rangle$ as described in the Supplemental Material [27], with $\Pi_{i, j}=\left|B_{i, j}\right\rangle\left\langle B_{i, j}\right|$ the projector on the specific basis element $\left|B_{i, j}\right\rangle$. All expectation values $\langle$.$\rangle are evaluated for the joint output state$ $\left(\mathcal{I}_{A} \otimes \mathcal{E}\right)\left(\left|\Phi^{+}\right\rangle\left\langle\Phi^{+}\right|\right)$and, using the normalization constraints among $a, b, c$, and $d$, it can be demonstrated that all probabilities $p_{i, j}$ depend only on two real parameters, $b$ and $d$. After collecting the measurement outcomes, the bound on $Q$ is then maximized over the three bases $B_{1}, B_{2}, B_{3}$, and by varying $b$ and $d$ :

$$
\begin{aligned}
Q_{D E T} & =\max _{i=1,2,3} \max _{b, d} Q_{D E T}\left(B_{i}, b, d\right) \\
& =S[\mathcal{E}(\rho)]-\min _{i=1,2,3} \min _{b, d} H\left[\vec{p}\left(B_{i}, b, d\right)\right]
\end{aligned}
$$

This last step is performed by classical processing of the measurement outcomes, from which the set of expectation values $\left\{\left\langle\mathbb{I} \otimes \sigma_{\alpha}\right\rangle,\left\langle\sigma_{\alpha} \otimes \mathbb{I}\right\rangle,\left\langle\sigma_{\alpha} \otimes \sigma_{\alpha}\right\rangle\right.$, $\alpha=x, y, x\}$ is obtained. Differently from a complete process tomography, we remark that we do not need to measure the six observables of the kind $\sigma_{\alpha} \otimes \sigma_{\beta}$ with $\alpha \neq \beta$ and, moreover, the bound is directly obtained from the measured expectations, without need of linear inversion and/or maximum likelihood technique. Let us also notice that the use of an entangled input state in our procedure is not mandatory. In fact, the ancilla is locally measured and this is equivalent to herald a single-photon state at the channel [28].

Experimental Procedure: We implemented the method using a SPDC source of high purity polarization 
entangled photons 29] schematically represented in Fig. 1. where the qubits were encoded as $\{|0\rangle \equiv|H\rangle,|1\rangle \equiv$ $|V\rangle\}$, with $|H\rangle(|V\rangle)$ the horizontal (vertical) polarization.

From a continuous wave laser pumping at $405 \mathrm{~nm}$ and bandwidth $<0.01 \mathrm{pm}$ we generate down-converted pairs of single photons at $810 \mathrm{~nm}$ and bandwidth $\approx 0.42 \mathrm{~nm}$, hence entangled in Bell states with a measured fidelity of $F_{\text {exp }}=0.979 \pm 0.011$, calculated as reported in 27] and using standard tomography analysis [30. The entanglement degree of the generated photons corresponds to an average concurrence value of $C_{e x p}=0.973 \pm 0.004$ [31.

The input state is ideally the maximally entangled state $\left|\Phi^{+}\right\rangle$. Due to experimental imperfections, the resulting state can be described by a Werner state $\rho_{W}=$ $\frac{4 F-1}{3}\left|\Phi^{+}\right\rangle\left\langle\Phi^{+}\right|+\frac{1-F}{3} I \otimes I$, where $F$ is the fidelity with respect to $\left|\Phi^{+}\right\rangle$. The derivation of new detectable bounds suited for bipartite input states which are affected by isotropic noise is reported in 27]. Such bounds will be used to compare our experimental results with the theoretical predictions.

The procedure was tested for the following types of noise: Amplitude Damping Channel (ADC), Phase Damping Channel (PDC), Depolarizing Channel (DC), and Pauli Channel (PC) 32, 33. As remarked above, we employ 3 versus 9 measurements on the output state, as in a usual process tomography.

The polarization based measurement is performed via a Quarter Wave Plate (QWP), a Half Wave Plate (HWP), and a Polarization Beam Splitter (PBS) located in both the reference (ancilla-A) and principal system (system-S) paths.

The expectation values $\left\langle\sigma_{\alpha} \otimes \sigma_{\alpha}\right\rangle$ (with $\alpha=x, y, z$ ) were obtained by taking every matrix element of $\sigma_{\alpha} \otimes \sigma_{\alpha}$ as a particular projection of the transmitted state ([27]), which was measured by integrating photon coincidences during a time interval of 5sec. Since only a set of 12 measurements of the state are needed ( 4 by each of the 3 observables), the $Q_{D E T}$ can be obtained in only 60 sec. See [27] for information about actual photon count rates and detection efficiencies.

An ADC for polarization qubits with probability $\gamma$ can be written as $\mathcal{E}(\rho)=K_{0} \rho K_{0}^{\dagger}+K_{1} \rho K_{1}^{\dagger}$, where $K_{0}=|H\rangle\langle H|+\sqrt{1-\gamma}| V\rangle\langle V|$ and $K_{1}=\sqrt{\gamma}|H\rangle\langle V|$. The experimental setup 34 consists in a Sagnac interferometer (SI) followed by a Mach-Zehnder interferometer (MZI), as shown in Fig $2 \mathrm{p}$, that allows us to perform the required noise operation. In the Sagnac loop, composed by a PBS and three mirrors, a HWP at 0 degree is put on the path of polarization $|H\rangle$, whereas a HWP at $\theta$ is put on the path of polarization $|V\rangle$. The MZI recombines the two outputs of the SI in a non-coherent superposition within a Beam Splitter (BS), thus effectively performing the damping operation, whose amount $\gamma$ depends on the angle $\theta$ 35.

A PDC for qubits with probability $p$ can be written as $\mathcal{E}(\rho)=\left(1-\frac{p}{2}\right) \rho+\frac{p}{2} \sigma_{z} \rho \sigma_{z}$. This map is achieved by using a sequence of two wave retarders: a HWP at 0 degree,
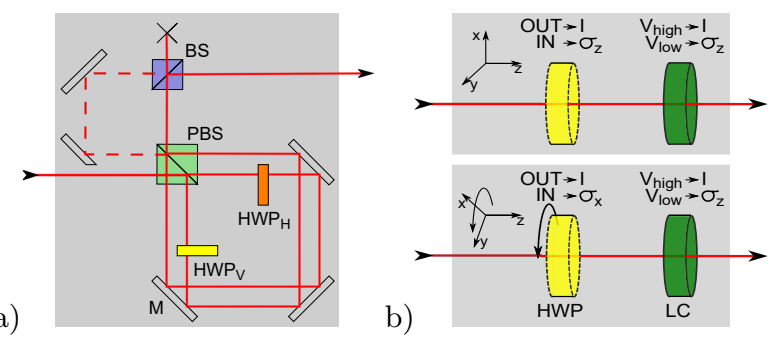

FIG. 2. Noisy Channels. a) Experimental scheme for a polarization ADC. Given a single input qubit $|\psi\rangle=\alpha|H\rangle+$ $\beta|V\rangle$, it uses two HWPs inside a SI to apply a rotation on $|V\rangle$, while $|H\rangle$ remains unrotated, achieving $K_{0}$ and $K_{1}$. The dashed line inside the MZI represents the rotated $\sqrt{\gamma}$ portion of $|V\rangle$. b)-top Experimental scheme for a PDC. It applies $\mathbb{I}$ and $\sigma_{z}$ over $|\psi\rangle$ by using an unrotated HWP and a variable voltage LC. b)-bottom: Experimental scheme for the PC and DC. They apply $\mathbb{I}, \sigma_{x}, \sigma_{y}$ and $\sigma_{z}$ over $|\psi\rangle$ by using a $45^{\circ}$-rotated HWP and the same LC.

which acts as $\sigma_{z}$, and an unrotated Liquid Crystal (LC), which acts as $\mathbb{I}$ or $\sigma_{z}$ depending on the applied voltage on the material [31. The system qubit is sent through the combination of this two optical elements, suffering a $\sigma_{z} \cdot \sigma_{z}=\mathbb{I}$ or $\mathbb{I} \cdot \sigma_{z}=\sigma_{z}$ operations, while the ancilla qubit remains untouched. In Fig. 2b-Top we show the schematic representation of the channel. The probabilities $P_{z}=\frac{p}{2}$ and $P_{\mathbb{I}}=1-P_{z}=1-\frac{p}{2}$ are applied in post-processing, by evaluating the ratios of photon coincidences from two separated experiments, $\sigma_{z}$ and $\mathbb{I}$ respectively. Therefore the total channel corresponds to a mixture of two operations constrained by $P_{z}+P_{\mathbb{I}}=1$.

A DC for qubits with probability $p$ can be written as $\mathcal{E}(\rho)=(1-p) \rho+\frac{p}{3}\left(\sigma_{x} \rho \sigma_{x}+\sigma_{y} \rho \sigma_{y}+\sigma_{z} \rho \sigma_{z}\right)$. The operations are achieved by the presence (absence) of a rotated HWP acting as $\sigma_{x}$ when it is on the optical path or acting as $\mathbb{I}$ if taken off from the path, and by an unrotated LC acting as $\mathbb{I}$ or $\sigma_{z}$, depending on the applied voltage. From the simultaneous actions of the HWP and the LC over $\rho$, the operations can be $\mathbb{I} \cdot \mathbb{I}=\mathbb{I}, \sigma_{z} \cdot \mathbb{I}=\sigma_{z}$, $\sigma_{z} \cdot \sigma_{x}=i \sigma_{y}$ and $\mathbb{I} \cdot \sigma_{x}=\sigma_{x}$. In Fig. 2p-Bottom we show the schematic representation of the channel. Analogously to the procedure followed for the PDC, the effective $\sigma_{z}$, $\sigma_{y}, \sigma_{x}$ and $\mathbb{I}$ operations were applied in four separated experiments, where $P_{z}=P_{y}=P_{x}=\frac{p}{3}$ and $P_{\mathbb{I}}=1-p$ were added in post-processing of the experimental outcomes.

The $\mathbf{P C}$ for qubits with probability $p$ can be written as $\mathcal{E}(\rho)=P_{\mathbb{I}} \rho+P_{x} \sigma_{x} \rho \sigma_{x}+P_{y} \sigma_{y} \rho \sigma_{y}+P_{z} \sigma_{z} \rho \sigma_{z}$ and has been implemented by the same procedure used for the DC. However, in this case there is no restriction on the choice of $P_{i}$ except for the condition $P_{\mathbb{I}}+P_{z}+P_{y}+P_{x}=1$, also valid for DC.

Results: In Fig. 3 we show the experimental measurements of $Q_{D E T}$ for a prepared ADC, PDC, DC and PC. The experimental values are in very good agreement with the theoretical predictions.

These results prove the effectiveness of the method, 
a)
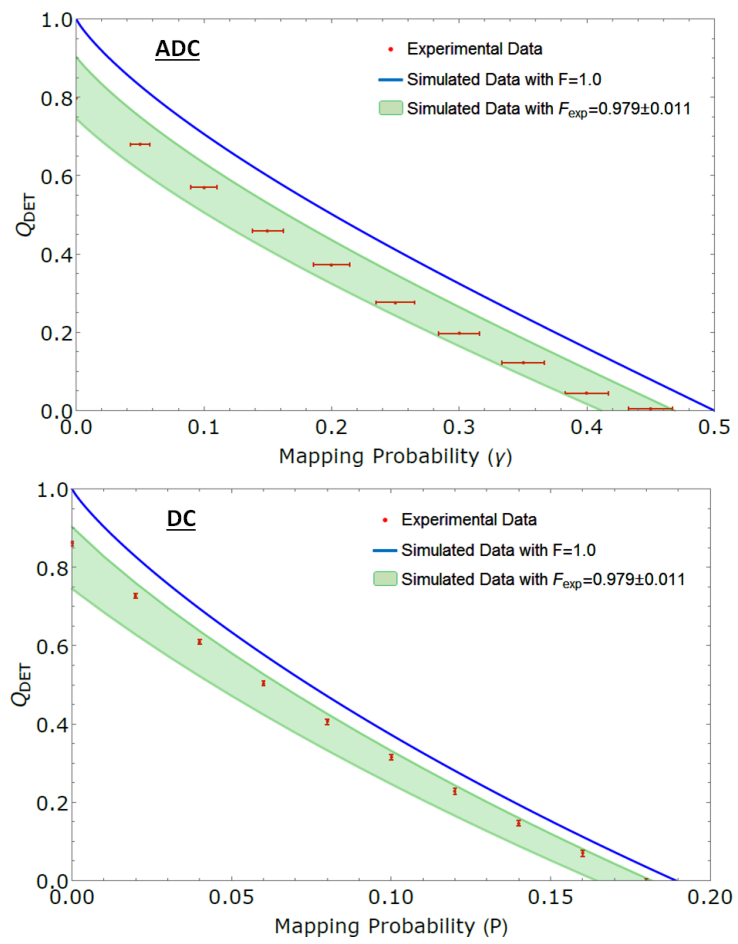

b)
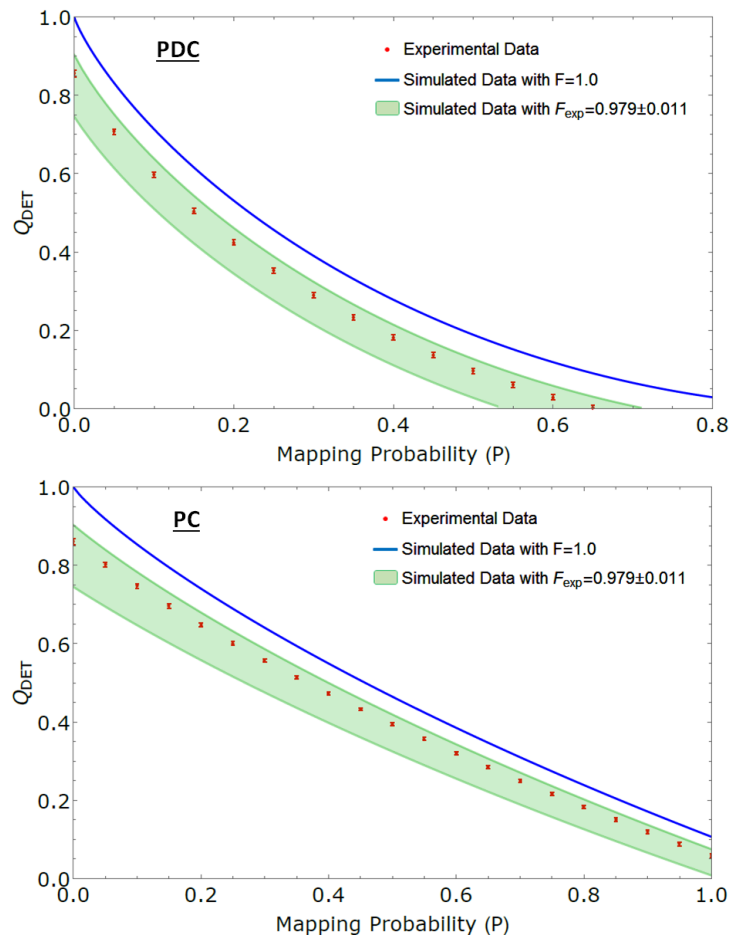

FIG. 3. Minimal bounds $Q_{D E T}$ to the quantum channel capacity. For the entire set of data, the red points represent the experimental values, the continuous blue line corresponds to the ideal simulation of a pure input state $\left|\Phi^{+}\right\rangle$with fidelity $F=1$. Green shaded areas correspond to a region of $Q_{D E T}$ for an input Werner state $\rho_{W}$ within one standard deviation of fidelity $F_{\text {exp }}=0.979 \pm 0.011$. a) ADC: $Q_{D E T}$ vs. the damping parameter $\gamma$. b) PDC: $Q_{D E T}$ vs. $p$ for the statistical mixture of $\mathbb{I}$ and $\sigma_{z}$, with probability vector $\vec{p}=\left\{P_{\mathbb{I}}, P_{x}, P_{y}, P_{z}\right\}=\left\{1-\frac{p}{2}, 0,0, \frac{p}{2}\right\}$. c) DC: $Q_{D E T}$ vs. $p$ for the balanced mixture of $\mathbb{I}$, $\sigma_{x}, \sigma_{y}$ and $\sigma_{z}$, with $\vec{p}=\left\{1-p, \frac{p}{3}, \frac{p}{3}, \frac{p}{3}\right\}$. d) PC: $Q_{D E T}$ vs. $p$ for the mixture of $\mathbb{I}, \sigma_{x}, \sigma_{y}$, and $\sigma_{z}$, with $\vec{p}=\left\{1-\frac{p}{6}, \frac{p}{12}, \frac{p}{18}, \frac{p}{36}\right\}$. The error bars on the mapping probability are significant only in the case a), where an uncertainty of 0.5 degrees in the rotation angle of $H W P_{V}(\gamma)$ was propagated, while this error was negligible in the cases b), c) and d). In the case a), the error bars on $Q_{D E T}$ were calculated from Poissonian statistics on one set of points, obtaining negligible values. In the case of b), c) and d), error bars were obtained from an average of at least 8 sets of points. (27] for more details).

which assesses efficiently the lower bound of $Q_{D E T}$ to the quantum capacity of noisy channels with the most common kinds of noise.

Moreover, the well known expressions for the ADC and PDC quantum capacities [5, 36] coincide with our detectable bound. The expected non-zero capacity for the ADC occurs when $\gamma<1 / 2$, while the certified experimental value was $\gamma<0.45$ (see Fig. 3 a). For the PDC, we obtained a non vanishing value of the $Q_{D E T}$ for values of $p$ up to 0.65 , whereas the ideal analytical expression is positive for any value of $p$ (see Fig. $3 \mathrm{p}$ )).

In the case of $\mathrm{DC}$, the procedure certifies a nonzero channel capacity for values of $p \leq 0.18$, while the best theoretical lower bound predicts a nonzero channel capacity up to $p=0.1892$ 37. Analogously, the technique certifies a nonzero channel capacity of the chosen PC for any value of $p$, similar to the ideal simulated channel.

Conclusions: We have performed an experiment to detect efficient lower bounds to the quantum capacity of qubit communication channels. Our technique does not require any prior knowledge of the quantum channel, and can be applied to any kind of unknown noise. The principal feature of the technique resides on the smaller number of measurement settings with respect to a full process tomography, and is in very good agreement with the theoretical prediction for the source we used. To the best of our knowledge this is the first experiment where the quantum capacity of a noisy channel is directly accessed. Furthermore, the detectable bounds we have provided give lower bounds to the private information and to the entanglement-assisted classical capacity, as emphasized in [23].

These results represent an important step toward an efficient experimental characterization of quantum channels such as those used in quantum cryptography, quantum teleportation, and quantum dense coding.

Acknowledgements: Á. Cuevas would like to thank the support from the Chilean agency CONICYT and to its PhD scholarships program. 


\section{Appendix: Supplemental Material}

\section{On the effect of noise of the input state in the experimental detection of quantum channel capacities.}

The theoretical results of Ref. [23], summarized in Eqs. (3-5) of the Letter, were derived under the assumption of sending a pure bipartite state at the input of the unknown channel. Realistically, as in the present experimental set-up, the generated input state will be affected by noise, thus producing a mixed state at the channel input.

Specifically, since our source indeed generates a maximally entangled state affected by isotropic noise, in the following, for arbitrary finite dimension $d$, we consider an isotropic noise map $\mathcal{N}$, leading to a bipartite mixed input state $\nu$ given by the convex combination of a maximally entangled state $\left|\Phi^{+}\right\rangle=\sum_{n=0}^{d-1}|n\rangle|n\rangle / \sqrt{d}$ and the totally mixed state $\frac{1}{d^{2}} I \otimes I$. Then, we can write

$$
\nu \equiv(I \otimes \mathcal{N})\left|\Phi^{+}\right\rangle\left\langle\Phi^{+}|=(\mathcal{N} \otimes I)| \Phi^{+}\right\rangle\left\langle\Phi^{+}\left|=\frac{d^{2} F-1}{d^{2}-1}\right| \Phi^{+}\right\rangle\left\langle\Phi^{+}\right|+\frac{1-F}{d^{2}-1} I \otimes I,
$$

in terms of the fidelity $\mathrm{F}$ with the ideal maximally entangled state, namely $F=\left\langle\Phi^{+}|\nu| \Phi^{+}\right\rangle$.

In the case of $d=2$ we have $\nu \rightarrow \rho_{W} \equiv \frac{4 F-1}{3}\left|\Phi^{+}\right\rangle\left\langle\Phi^{+}\right|+\frac{1-F}{3} I \otimes I$, also known as Werner state. In our work, the experimental input density matrix $\nu$ is calculated by a Mathematica algorithm based on two steps. In the first one, a preliminary matrix $\nu^{\prime}$ is computed by considering the registered photon coincidences of 36 suitable plates rotations as projections of the experimental state. In the second step, the algorithm imposes positivity and forces $\nu^{\prime}$ to approach a legitimate positive matrix $\nu$. This is done by a Maximum Likelihood Maximization process, based on Poisson statistics, resulting in a physical version of $\nu$, which is now a positive matrix with unit trace 38. Then, the fidelity $F$ is obtained by the straightforward evaluation of $\left\langle\Phi^{+}|\nu| \Phi^{+}\right\rangle$.

As long as the noise map $\mathcal{N}$ acts on maximally entangled states, its action can be equivalently ascribed to the system or the ancilla qudit. For this reason the use of a noisy bipartite state as in Eq. A.1) for our detection protocol is equivalent to having a perfect input $\left|\Phi^{+}\right\rangle$along with a quantum measurement degraded by the isotropic dual map $\mathcal{N}^{\vee}$ (the map in the Heisenberg picture). In fact, for any measurement basis $\left\{\left|\Phi_{i}\right\rangle\right\}$ the reconstructed probabilities are

$$
\begin{aligned}
p_{i} & =\operatorname{Tr}\left[\left(\mathcal{I}_{A} \otimes \mathcal{E}\right)\left(\mathcal{I}_{A} \otimes \mathcal{N}\right)\left(\left|\Phi^{+}\right\rangle\left\langle\Phi^{+}\right|\right)\left|\Phi_{i}\right\rangle\left\langle\Phi_{i}\right|\right]=\operatorname{Tr}\left[\left(\mathcal{I}_{A} \otimes \mathcal{E}\right)\left(\left|\Phi^{+}\right\rangle\left\langle\Phi^{+}\right|\right)\left(\mathcal{N}^{\vee} \otimes \mathcal{I}\right)\left(\left|\Phi_{i}\right\rangle\left\langle\Phi_{i}\right|\right)\right] \\
& =\operatorname{Tr}\left[\left(\mathcal{I}_{A} \otimes \mathcal{E}\right)\left(\left|\Phi^{+}\right\rangle\left\langle\Phi^{+}\right|\right) \Pi_{i}\right]
\end{aligned}
$$

where

$$
\Pi_{i} \equiv\left(\mathcal{N}^{\vee} \otimes \mathcal{I}\right)\left(\left|\Phi_{i}\right\rangle\left\langle\Phi_{i}\right|\right)
$$

is generally the element of a POVM. Then, in order to take into account the effect of noise, we need to generalize the bound of Ref. 23]

$$
S_{e}(\rho, \mathcal{E}) \leq H(\vec{p})
$$

to the case of a probability vector $\vec{p}$ pertaining to an arbitrary $\operatorname{POVM}\left\{\Pi_{i}\right\}$ for the tensor product of the reference and system Hilbert spaces, where

$$
p_{i}=\operatorname{Tr}\left[\left(\mathcal{I}_{A} \otimes \mathcal{E}\right)\left(\left|\Psi_{\rho}\right\rangle\left\langle\Psi_{\rho}\right|\right) \Pi_{i}\right]
$$

In the following we provide such a generalization. Let us consider a density matrix $\sigma$, along with its spectral decomposition $\sigma=\sum_{j} s_{j}\left|\phi_{j}\right\rangle\left\langle\phi_{j}\right|$, and let us define the conditional probability $p(i \mid j)=\left\langle\phi_{j}\left|\Pi_{i}\right| \phi_{j}\right\rangle$. Clearly, one has $p_{i} \equiv \operatorname{Tr}\left[\sigma \Pi_{i}\right]=\sum_{j} s_{j} p(i \mid j)$. Then,

$$
\begin{aligned}
S(\sigma)-H(\vec{p}) & =\sum_{i} p_{i} \log _{2} p_{i}-\sum_{j} s_{j} \log _{2} s_{j}=\sum_{i, j} s_{j} p(i \mid j)\left(\log _{2} p_{i}-\log _{2} s_{j}\right) \\
& \leq \log _{2}\left(\sum_{i, j} s_{j} p(i \mid j) \frac{p_{i}}{s_{j}}\right)=\log _{2} \vec{r} \cdot \vec{p}
\end{aligned}
$$

where we used Jensen's inequality, and defined $\vec{r}$ with vector components $r_{i}=\sum_{j}\left\langle\phi_{j}\left|\Pi_{i}\right| \phi_{j}\right\rangle$. Upon choosing $\sigma=\left(\mathcal{I}_{R} \otimes \mathcal{E}\right)\left(\left|\Psi_{\rho}\right\rangle\left\langle\Psi_{\rho}\right|\right)$, one obtains the more general bound

$$
S_{e}(\rho, \mathcal{E}) \equiv S(\sigma) \leq H(\vec{p})+\log _{2} \vec{t} \cdot \vec{p}
$$


with $p_{i}$ as in Eq. A.5 and $t_{i} \equiv \operatorname{Tr}\left[\Pi_{i}\right] \geq r_{i}$. Then, the detected quantum capacity $Q_{D E T}$ in Eq. (4) of the Letter is simply replaced with

$$
Q_{D E T}=S[\mathcal{E}(\rho)]-H(\vec{p})-\log _{2} \vec{t} \cdot \vec{p},
$$

in terms of the noisy reconstructed probabilities of Eq. A.2).

Notice now that for any unital noise map (as the isotropic-noise one), the dual map is trace-preserving. Hence, if $\Pi_{i}$ is of the form as in Eq. A.3, one has $t_{i} \equiv \operatorname{Tr}\left[\Pi_{i}\right]=1$. Then, the last term in A.8 vanishes, namely $\log _{2} \vec{t} \cdot \vec{p}=0$.

Let us see the effect of noise on the quantum capacity detection for specific channels.

1) Amplitude Damping Channel (ADC) for qubits

$$
\mathcal{E}(\rho)=K_{0} \rho K_{0}^{\dagger}+K_{1} \rho K_{1}^{\dagger},
$$

where $K_{0}=|0\rangle\langle 0|+\sqrt{1-\gamma}| 1\rangle\langle 1|$ and $K_{1}=\sqrt{\gamma}|0\rangle\langle 1|$.

For an input state as in Eq. A.1), the bipartite output is given by

$$
\begin{aligned}
\left(\mathcal{I}_{A} \otimes \mathcal{E}\right) \nu & =\left(c_{1} \gamma_{+}+c_{2}\right)\left|\Phi^{+}\right\rangle\left\langle\Phi^{+}\left|+\left(c_{1} \gamma_{-}+c_{2}\right)\right| \Phi^{-}\right\rangle\left\langle\Phi^{-}\right|+\frac{\gamma}{4}\left(\left|\Phi^{+}\right\rangle\left\langle\Phi^{-}|+| \Phi^{-}\right\rangle\left\langle\Phi^{+}\right|\right) \\
& +\frac{1}{4}\left(\gamma c_{1}+4 c_{2}\right)\left(\left|\Psi^{+}\right\rangle\left\langle\Psi^{+}|+| \Psi^{-}\right\rangle\left\langle\Psi^{-}\right|\right)-\frac{\gamma}{4}\left(\left|\Psi^{+}\right\rangle\left\langle\Psi^{-}|+| \Psi^{-}\right\rangle\left\langle\Psi^{+}\right|\right),
\end{aligned}
$$

with $\gamma_{ \pm}=\frac{1}{4}(1 \pm \sqrt{1-\gamma})^{2}, c_{1}=(4 F-1) / 3$, and $c_{2}=(1-F) / 3$. The reduced output state is given by $\mathcal{E}\left(\frac{I}{2}\right)=$ $\frac{1}{2}\left(I+\gamma \sigma_{z}\right)$, hence it has von Neumann entropy $S\left[\mathcal{E}\left(\frac{I}{2}\right)\right]=H_{2}\left(\frac{1-\gamma}{2}\right)$. By performing the local measurement of $\sigma_{x} \otimes \sigma_{x}, \sigma_{y} \otimes \sigma_{y}$, and $\sigma_{z} \otimes \sigma_{z}$, estimating the von Neumann entropy $S\left[\mathcal{E}\left(\frac{I}{2}\right)\right]$, and optimising $\vec{p}$, one can detect the bound

$$
Q \geq Q_{D E T}=H_{2}\left(\frac{1-\gamma}{2}\right)-H(\vec{p}),
$$

where the optimal vector of probabilities is given by

$$
\begin{aligned}
\vec{p}= & \left(\frac{2+4 F(1-\gamma)+\gamma+\sqrt{4(1-4 F)^{2}(1-\gamma)+9 \gamma^{2}}}{12}, \frac{2+4 F(1-\gamma)+\gamma-\sqrt{4(1-4 F)^{2}(1-\gamma)+9 \gamma^{2}}}{12},\right. \\
& \left.\frac{(1-F)(1-\gamma)}{3}, \frac{2+\gamma-2 F(1-\gamma)}{6}\right) .
\end{aligned}
$$

Such probabilities correspond to the optimal basis

$$
\left\{a\left|\Phi^{+}\right\rangle+b\left|\Phi^{-}\right\rangle,-b\left|\Phi^{+}\right\rangle+a\left|\Phi^{-}\right\rangle, \frac{1}{\sqrt{2}}\left(\left|\Psi^{+}\right\rangle+\left|\Psi^{-}\right\rangle\right) \equiv|01\rangle, \frac{1}{\sqrt{2}}\left(\left|\Psi^{+}\right\rangle-\left|\Psi^{-}\right\rangle\right) \equiv|10\rangle\right\},
$$

where

$$
a=\sqrt{\frac{\cosh \eta+1}{2 \cosh \eta}}, \quad b=\sqrt{\frac{\cosh \eta-1}{2 \cosh \eta}},
$$

with $\eta \equiv \operatorname{arcsinh}\left(\frac{3 \gamma}{2(4 F-1) \sqrt{1-\gamma}}\right)$.

The case of amplitude damping channel is a relevant example showing that the Bell basis can be sub-optimal for the quantum capacity certification.

2) For the Phase Damping Channel (PDC) for qubits

$$
\mathcal{E}(\rho)=\left(1-\frac{p}{2}\right) \rho+\frac{p}{2} \sigma_{z} \rho \sigma_{z},
$$

one has

$$
Q=Q_{1}=1-H_{2}\left(\frac{p}{2}\right) \geq Q_{D E T}=1-H(\vec{p}),
$$

where the vector of probabilities $\vec{p}$ is given by

$$
\vec{p}=\left\{\left(1-\frac{p}{2}\right) F+\frac{p}{2} \frac{1-F}{3}, \frac{p}{2} F+\left(1-\frac{p}{2}\right) \frac{1-F}{3}, \frac{1-F}{3}, \frac{1-F}{3}\right\},
$$


which corresponds to the Bell basis.

3) For the Depolarizing Channel (DC) in dimension $d$

$$
\mathcal{E}(\rho)=\left(1-p \frac{d^{2}}{d^{2}-1}\right) \rho+p \frac{d^{2}}{d^{2}-1} \frac{I}{d}
$$

the detectable bound is now

$$
Q \geq Q_{D E T}=\log _{2} d-H_{2}\left(p^{\prime}\right)-p^{\prime} \log _{2}\left(d^{2}-1\right)
$$

where

$$
p^{\prime}=\frac{\left.d^{2}[1-F(1-p)]+F-p-1\right]}{d^{2}-1}
$$

For qubits

$$
Q \geq Q_{D E T}=1-H_{2}\left(p^{\prime}\right)-p^{\prime} \log _{2} 3,
$$

with $p^{\prime}=(1-F)+\frac{p}{3}(4 F-1)$. The reconstructed probabilities for the Bell basis $\left\{\left|\Phi^{+}\right\rangle,\left|\Phi^{-}\right\rangle,\left|\Psi^{+}\right\rangle,\left|\Psi^{-}\right\rangle\right\}$correspond to $\left\{1-p^{\prime}, \frac{p^{\prime}}{3}, \frac{p^{\prime}}{3}, \frac{p^{\prime}}{3}\right\}$.

4) For a Pauli Channel (PC) in dimension $d$

$$
\mathcal{E}(\rho)=\sum_{m, n=0}^{d-1} p_{m n} U_{m n} \rho U_{m n}^{\dagger},
$$

with $U_{m n}=\sum_{k=0}^{d-1} e^{\frac{2 \pi i}{d} k m}|k\rangle\langle(k+n) \bmod d|$, one has

$$
Q \geq Q_{D E T}=\log _{2} d-H\left(\vec{p}^{\prime}\right),
$$

where $\vec{p}^{\prime}$ is the $d^{2}$-dimensional vector of probabilities pertaining to the generalised Bell projectors, whose components are given by

$$
p_{m n}^{\prime}=\frac{1}{d^{2}-1}\left[\left(d^{2} F-1\right) p_{m n}+1-F\right] .
$$

For the qubit case, $\mathcal{E}(\rho)=\sum_{i=0}^{3} p_{i} \sigma_{i} \rho \sigma_{i}$, and $p_{i}^{\prime}=\frac{1}{3}\left[(4 F-1) p_{i}+1-F\right]$. 


\section{Experimental Expectation Values}

The expanded expressions of the probability vectors $p_{i, j}=\left\langle\Pi_{i, j}\right\rangle$ used in our experiment obey the following form, described here for $B_{1}$ :

$$
\begin{aligned}
p_{1,1} & =\left\langle\left(a\left|\Phi^{+}\right\rangle+b\left|\Phi^{-}\right\rangle\right)\left(a\left\langle\Phi^{+} \mid+b\left\langle\Phi^{-}\right|\right)\right\rangle\right. \\
& =\frac{1}{4}\left(\langle\mathbb{I} \otimes \mathbb{I}\rangle+\left\langle\sigma_{z} \otimes \sigma_{z}\right\rangle\right)+\frac{a b}{2}\left(\left\langle\sigma_{z} \otimes \mathbb{I}\right\rangle+\left\langle\mathbb{I} \otimes \sigma_{z}\right\rangle\right)+\frac{a^{2}-b^{2}}{4}\left(\left\langle\sigma_{x} \otimes \sigma_{x}\right\rangle-\left\langle\sigma_{y} \otimes \sigma_{y}\right\rangle\right), \\
p_{1,2} & =\left\langle\left(-b\left|\Phi^{+}\right\rangle+a\left|\Phi^{-}\right\rangle\right)\left(-b\left\langle\Phi^{+} \mid+a\left\langle\Phi^{-}\right|\right)\right\rangle\right. \\
& =\frac{1}{4}\left(\langle\mathbb{I} \otimes \mathbb{I}\rangle+\left\langle\sigma_{z} \otimes \sigma_{z}\right\rangle\right)-\frac{a b}{2}\left(\left\langle\sigma_{z} \otimes \mathbb{I}\right\rangle+\left\langle\mathbb{I} \otimes \sigma_{z}\right\rangle\right)-\frac{a^{2}-b^{2}}{4}\left(\left\langle\sigma_{x} \otimes \sigma_{x}\right\rangle-\left\langle\sigma_{y} \otimes \sigma_{y}\right\rangle\right), \\
p_{1,3} & =\left\langle\left(c\left|\Psi^{+}\right\rangle+d\left|\Psi^{-}\right\rangle\right)\left(c\left\langle\Psi^{+} \mid+d\left\langle\Psi^{-}\right|\right)\right\rangle\right. \\
& =\frac{1}{4}\left(\langle\mathbb{I} \otimes \mathbb{I}\rangle-\left\langle\sigma_{z} \otimes \sigma_{z}\right\rangle\right)+\frac{c d}{2}\left(\left\langle\sigma_{z} \otimes \mathbb{I}\right\rangle-\left\langle\mathbb{I} \otimes \sigma_{z}\right\rangle\right)+\frac{c^{2}-d^{2}}{4}\left(\left\langle\sigma_{x} \otimes \sigma_{x}\right\rangle+\left\langle\sigma_{y} \otimes \sigma_{y}\right\rangle\right), \\
p_{1,4} & =\left\langle\left(-d\left|\Psi^{+}\right\rangle+c\left|\Psi^{-}\right\rangle\right)\left(-d\left\langle\Psi^{+} \mid+c\left\langle\Psi^{-}\right|\right)\right\rangle\right. \\
& =\frac{1}{4}\left(\langle\mathbb{I} \otimes \mathbb{I}\rangle-\left\langle\sigma_{z} \otimes \sigma_{z}\right\rangle\right)-\frac{c d}{2}\left(\left\langle\sigma_{z} \otimes \mathbb{I}\right\rangle-\left\langle\mathbb{I} \otimes \sigma_{z}\right\rangle\right)-\frac{c^{2}-d^{2}}{4}\left(\left\langle\sigma_{x} \otimes \sigma_{x}\right\rangle+\left\langle\sigma_{y} \otimes \sigma_{y}\right\rangle\right)
\end{aligned}
$$

The rest of the probability vectors, $\vec{p}_{2, j}$ and $\vec{p}_{3, j}$, can be calculated analogously.

Our protocol only needs the following expectation values of observables on the joint ancilla-system state

$$
\begin{aligned}
& \left\langle\sigma_{z} \otimes \sigma_{z}\right\rangle=\frac{1}{N_{z}} C C(|H\rangle\langle H|\otimes| H\rangle\langle H|-| H\rangle\langle H|\otimes| V\rangle\langle V|-| V\rangle\langle V|\otimes| H\rangle\langle H|+| V\rangle\langle V|\otimes| V\rangle\langle V|), \\
& \left\langle\sigma_{y} \otimes \sigma_{y}\right\rangle=\frac{1}{N_{y}} C C(|R\rangle\langle R|\otimes| R\rangle\langle R|-| R\rangle\langle R|\otimes| L\rangle\langle L|-| L\rangle\langle L|\otimes| R\rangle\langle R|+| L\rangle\langle L|\otimes| L\rangle\langle L|), \\
& \left\langle\sigma_{x} \otimes \sigma_{x}\right\rangle=\frac{1}{N_{x}} C C(|+\rangle\langle+|\otimes|+\rangle\langle+|-|+\rangle\langle+|\otimes|-\rangle\langle-|-|-\rangle\langle-|\otimes|+\rangle\langle+|+|-\rangle\langle-|\otimes|-\rangle\langle-|), \\
& \langle\mathbb{I} \otimes \mathbb{I}\rangle=\frac{1}{N_{z}} C C(|H\rangle\langle H|\otimes| H\rangle\langle H|+| H\rangle\langle H|\otimes| V\rangle\langle V|+| V\rangle\langle V|\otimes| H\rangle\langle H|+| V\rangle\langle V|\otimes| V\rangle\langle V|), \\
& \left\langle\sigma_{z} \otimes \mathbb{I}\right\rangle=\frac{1}{N_{z}} C C(|H\rangle\langle H|\otimes| H\rangle\langle H|+| H\rangle\langle H|\otimes| V\rangle\langle V|-| V\rangle\langle V|\otimes| H\rangle\langle H|-| V\rangle\langle V|\otimes| V\rangle\langle V|), \\
& \left\langle\sigma_{y} \otimes \mathbb{I}\right\rangle=\frac{1}{N_{y}} C C(|R\rangle\langle R|\otimes| R\rangle\langle R|+| R\rangle\langle R|\otimes| L\rangle\langle L|-| L\rangle\langle L|\otimes| R\rangle\langle R|-| L\rangle\langle L|\otimes| L\rangle\langle L|), \\
& \left\langle\sigma_{x} \otimes \mathbb{I}\right\rangle=\frac{1}{N_{x}} C C(|+\rangle\langle+|\otimes|+\rangle\langle+|+|+\rangle\langle+|\otimes|-\rangle\langle-|-|-\rangle\langle-|\otimes|+\rangle\langle+|-|-\rangle\langle-|\otimes|-\rangle\langle-|), \\
& \left\langle\mathbb{I} \otimes \sigma_{z}\right\rangle=\frac{1}{N_{z}} C C(|H\rangle\langle H|\otimes| H\rangle\langle H|-| H\rangle\langle H|\otimes| V\rangle\langle V|+| V\rangle\langle V|\otimes| H\rangle\langle H|-| V\rangle\langle V|\otimes| V\rangle\langle V|), \\
& \left\langle\mathbb{I} \otimes \sigma_{y}\right\rangle=\frac{1}{N_{y}} C C(|R\rangle\langle R|\otimes| R\rangle\langle R|-| R\rangle\langle R|\otimes| L\rangle\langle L|+| L\rangle\langle L|\otimes| R\rangle\langle R|-| L\rangle\langle L|\otimes| L\rangle\langle L|), \\
& \left\langle\mathbb{I} \otimes \sigma_{x}\right\rangle=\frac{1}{N_{x}} C C(|+\rangle\langle+|\otimes|+\rangle\langle+|-|+\rangle\langle+|\otimes|-\rangle\langle-|+|-\rangle\langle-|\otimes|+\rangle\langle+|-|-\rangle\langle-|\otimes|-\rangle\langle-|),
\end{aligned}
$$

with

$$
\begin{aligned}
& N_{z}=C C(|H\rangle\langle H|\otimes| H\rangle\langle H|+| H\rangle\langle H|\otimes| V\rangle\langle V|+| V\rangle\langle V|\otimes| H\rangle\langle H|+| V\rangle\langle V|\otimes| V\rangle\langle V|), \\
& N_{y}=C C(|R\rangle\langle R|\otimes| R\rangle\langle R|+| R\rangle\langle R|\otimes| L\rangle\langle L|+| L\rangle\langle L|\otimes| R\rangle\langle R|+| L\rangle\langle L|\otimes| L\rangle\langle L|), \\
& N_{x}=C C(|+\rangle\langle+|\otimes|+\rangle\langle+|+|+\rangle\langle+|\otimes|-\rangle\langle-|+|-\rangle\langle-|\otimes|+\rangle\langle+|+|-\rangle\langle-|\otimes|-\rangle\langle-|) .
\end{aligned}
$$

In the above equations $C C(|i\rangle\langle i|\otimes| j\rangle\langle j|)$ denotes the coincident photon detections of the state within a time window, associated to the projection on the state $|i\rangle \otimes|j\rangle$ of the ancilla and system qubits, with $i, j=H, V, L, R,+,-$. The different bases are characterized as logical $\{|H\rangle,|V\rangle\}$, circular $\left\{|L\rangle=\frac{1}{\sqrt{2}}(|H\rangle+i|V\rangle),|R\rangle=\frac{1}{\sqrt{2}}(|H\rangle-i|V\rangle)\right\}$, or diagonal $\left\{|+\rangle=\frac{1}{\sqrt{2}}(|H\rangle+|V\rangle),|-\rangle=\frac{1}{\sqrt{2}}(|H\rangle-|V\rangle)\right\}$, while $N_{z}, N_{y}$, and $N_{x}$ are the normalization factors also expressed in terms of coincidences.

According to the above equations, all the expectation values are obtained just by the measurement of 12 polarization projections of the state ( 4 by each of the 3 observables), which makes the process efficient in terms registration 
and analysis of data, given the reduced number of operations compared with a standard process tomography. Equations A.33 A.35 are equivalent to take a partial trace of the S-qubit, and measure exclusively the A-qubit by triggering the coincidences with the detection of S. Similarly, equations A.36 A.38 are equivalent to take a partial trace of the A-qubit, and measure exclusively the S-qubit by triggering the coincidences with the detection of A.

\section{Photon Count Rates and Detection Efficiencies}

The overall detection efficiency of single photons in the system or ancilla modes of our experiment is given approximately by

$$
\epsilon_{\text {overall }} \approx \epsilon_{\text {source }} \cdot \epsilon_{\text {exp }} \cdot \epsilon_{\text {tomo }} \cdot \epsilon_{S M F} \cdot \epsilon_{A P D}
$$

Where $\epsilon_{\text {source }} \approx 95 \%$ is the transmissivity of down converted photons through the optical elements of the quantum source, $\epsilon_{\exp }$ is the transmissivity of the tested channel, $\epsilon_{\text {tomo }} \approx 99 \%$ is the transmissivity of the tomography optics, $\epsilon_{S M F} \approx 73 \%$ is the coupling efficiency of photons within a single mode optic fiber (SMF) and $\epsilon_{A P D} \approx 70 \%$ is the quantum efficiency of the Avalanche Photodetectors (APDs).

The system-S photons of the source were transmitted to the tested channels through a polarization compensated SMF link. Then, the effective efficiency of any kind of channel was composed by

$$
\epsilon_{\text {exp }} \approx \epsilon_{S M F} \cdot \epsilon_{\text {channel }}
$$

Where $\epsilon_{\text {channel }}$ corresponds to transmissivity of each bulk channel, ranging from $\approx 98 \%$ in the case of PDC, DC and $\mathrm{PC}$, to $\approx 60 \%$ for the ADC. Thus, without testing any kind of channel inside the mode $\left(\epsilon_{e x p}=1\right)$ the associated overall detection efficiency for single photons was $\epsilon_{\text {overall }} \approx 48 \%$. In the case of PDC, DC or PC the overall detection efficiency was $\approx 34 \%$, and for ADC was $\approx 21 \%$.

Considering $2.5 \mathrm{~mW}$ of laser pumping power and the overall single photon efficiency $\epsilon_{\text {overall }} \approx 48 \%$ from the SPDC process to the single photon detection in two synchronized APDs within a time window of $6 \mathrm{~ns}$, we get an effective source generation of nearly $C_{S}=C_{A}=375000 \frac{\text { single photons }}{\mathrm{sec}}$ in the S-A modes and $C_{S A}=60000 \frac{\text { coincident photons }}{\mathrm{sec}}$ without the presence of any channel on $\mathrm{S}$. These values corresponds to a heralding efficiency of

$$
\eta=\frac{C_{S A}}{\sqrt{C_{S} \cdot C_{A}}} \approx 16 \%
$$

From the above quantities it is also possible to calculate the real number of photons created by the source, right after the SPDC generation, even if we don't have access to them. To estimate that quantity we assume that $C_{S}=C_{A}$, then the source output counts will be

$$
\begin{aligned}
C_{S, \text { Source }} & =\frac{C_{S}}{\epsilon_{\text {overall }}} \approx 781000 \frac{\text { single photons }}{\text { sec }} \\
C_{S A, \text { Source }} & =\frac{C_{S A}}{\left(\epsilon_{\text {overall }}\right)^{2}} \approx 260000 \frac{\text { coincident photons }}{\text { sec }}
\end{aligned}
$$

The registered dark counts were $<500 \frac{\text { single photons }}{\text { sec }}$, and an accidental coincidence rate $<5 \%$ was evaluated.

\section{Experimental Error Analysis}

Each experimental error bar seen in our results originates from three main contributions:

1. Poissonian statistics on the coincidence photons counts, which is propagated using the a Monte Carlo simulation around the mean coincidence values registered in 5 seconds of integration.

2. The experimental channels can have systematic errors due an imperfect balance between the internal maps, or also random errors as the propagation of the uncertainty in the rotation of the HWP and LC.

3. Each experimental point is a mean of a different number of repetitions of the same experiment, so that we considered the standard deviation as a contribution to the random error. 
- For the ADC: The principal contribution to the total error originates from the uncertainty of the dumping preparation, namely the rotation of the $H W P_{V}(\varphi)=\left(\begin{array}{cc}\cos (2 \varphi) & -\sin (2 \varphi) \\ -\sin (2 \varphi) & -\cos (2 \varphi)\end{array}\right)$. In this case, there is a direct connection of the damping to the angle $\varphi$ of the plate through the equation

$$
\varphi=\frac{\arccos (-\sqrt{1-\gamma})}{2}
$$

Given the non-linearity of dumping, an uncertainty of $\Delta=0.5$ [degrees] can be very sensitive to any change.

Any other source of error, including the Poissonian distribution of the counts and its proper propagation is practically negligible compared with the propagation of $\Delta$. Thus the presentation of the $Q_{D e t}$ under the action of an ADC only have effective error bars in the x-axis.

- For the PC: Unlike the ADC, the PC is achieved by the combination of four different experiments $\left(\mathbb{I}, \sigma_{x}, \sigma_{y}, \sigma_{z}\right)$, which are prepared separately as a combinations of one HWP and one LC.

Even if we have more than one optical element with uncertainty $\Delta$, its error propagation represents a very small contribution to the total error. For example, let's consider a more general channel $\Lambda(\rho)=\sum_{i=0}^{3} p_{i} A_{i} \rho A_{i}^{\dagger}$ with $p_{0}+p_{1}+p_{2}+p_{3}=1$, and where each $A_{i}$ map is composed by two LCs, which have an effective operation of $\Gamma_{\text {low }}(\varphi)=\left(\begin{array}{cc}\cos (2 \varphi) & -\sin (2 \varphi) \\ -\sin (2 \varphi) & -\cos (2 \varphi)\end{array}\right)$ for a low tension value, and $\Gamma_{\text {high }}=\mathbb{I}$ for high tension values.

If we want to reproduce the imprecise Pauli channel, namely with an uncertainty of $\Delta$ for every rotation dependence of the LC's, we get that

$$
\begin{aligned}
\mathbb{I}=\sigma_{z} \circ \sigma_{z} \text { is achieved by } A_{0} & =\Gamma_{\text {low }}(0 \pm \Delta) \circ \Gamma_{\text {low }}(0 \pm \Delta) \\
\sigma_{x}=\sigma_{x} \circ \mathbb{I} \text { is achieved by } A_{1} & =\Gamma_{\text {low }}(\pi / 2 \pm \Delta) \circ \Gamma_{\text {high }} \\
\sigma_{y}=i \sigma_{x} \circ \sigma_{z} \text { is achieved by } A_{2} & =\Gamma_{\text {low }}(\pi / 2 \pm \Delta) \circ \Gamma_{\text {low }}(0 \pm \Delta) \\
\sigma_{z}=\mathbb{I} \circ \sigma_{z} \text { is achieved by } A_{3} & =\Gamma_{\text {high }} \circ \Gamma_{\text {low }}(0 \pm \Delta)
\end{aligned}
$$

It is not difficult to verify that $\Lambda$ transforms the state $\rho$ almost in the same way for any propagation of errors within $\Delta$. Then, $\Lambda$ applies the same degree of decoherence to $\rho$ for a propagated error within $\Delta$. The direct consequence of this fact is a negligible error contribution from the angle uncertainty to the final quantum channel capacity.

In this kind of channels the principal error contribution originates from the combination of state measurements obtained in different experiments, because of unavoidable photon counts fluctuations. Any other source of error, including the Poissonian distribution of the counts and its proper propagation is negligible. Thus, the presentation of the $Q_{D E T}$ under the action of a PC (and also for DC and PDC) only have effective error bars in the y-axis.

[1] S. Lloyd, Phys. Rev. A 55, 1613 (1997).

[2] H. Barnum, M. A. Nielsen, and B. Schumacher, Phys. Rev. A 57, 4153 (1998).

[3] I. Devetak, IEEE Trans. Inf. Theory 51, 44 (2003).

[4] P. Hayden, M. Horodecki, A. Winter, and J. Yard, Open Sys. Inf. Dyn. 15, 7 (2008).

[5] I. Devetak and P. Shor, Comm. Math. Phys. 256, 287 (2005).

[6] J. Yard, I. Devetak, and P. Hayden, IEEE Trans. Inf. Theory 54, 3091 (2008).

[7] T. S. Cubitt, M. Ruskai, and G. Smith, J. Math. Phys. 49, 102104 (2008).

[8] I. L. Chuang and M. A. Nielsen, Journal of Modern Optics 44, 2455 (1997).

[9] J. F. Poyatos, J. I. Cirac, and P. Zoller, Phys. Rev. Lett. 78, 390 (1997).

[10] M. F. Sacchi, Phys. Rev. A 63, 054104 (2001).

[11] G. M. D'Ariano and P. Lo Presti, Phys. Rev. Lett. 86, 4195 (2001).

[12] J. Altepeter, D. Branning, E. Jeffrey, T. Wei, P. Kwiat, R. Thew, J. OBrien, M. Nielsen, and A. White, Phys. Rev. Lett. 90, 193601 (2003).

[13] J. L. O’Brien, G. J. Pryde, A. Gilchrist, D. F. V. James, N. K. Langford, T. C. Ralph, and A. G. White, Phys. Rev. Lett. 93, 080502 (2004).

[14] S. H. Myrskog, J. K. Fox, M. W. Mitchell, and A. M. Steinberg, Phys. Rev. A 72, 013615 (2005). 
[15] M. Riebe, K. Kim, P. Schindler, T. Monz, P. O. Schmidt, T. K. Körber, W. Hänsel, H. Häffner, C. F. Roos, and R. Blatt, Phys. Rev. Lett. 97, 220407 (2006).

[16] M. Mohseni, A. T. Rezakhani, and D. A. Lidar, Phys. Rev. A 77, 032322 (2008).

[17] I. Bongioanni, L. Sansoni, F. Sciarrino, G. Vallone, and P. Mataloni, Phys. Rev. A 82, 042307 (2010).

[18] Yoav Sagi, Ido Almog, and Nir Davidson, Phys. Rev. Lett. 105, 053201 (2010).

[19] O. Gühne, P. Hyllus, D. Bruß, A. Ekert, M. Lewenstein, C. Macchiavello, and A. Sanpera, Phys. Rev. A 66, 062305 (2002).

[20] G. M. D'Ariano, M. G. A. Paris, and M. F. Sacchi, Phys. Rev. A 62, 023815 (2000).

[21] C. Macchiavello and M. Rossi, Phys. Rev. A 88, 042335 (2013); A. Orieux, L. Sansoni, M. Persechino, P. Mataloni, M. Rossi, and C. Macchiavello, Phys. Rev. Lett. 111, 220501 (2013).

[22] D. Chruściński, C. Macchiavello, and S. Maniscalco, Phys. Rev. Lett. 118, 080404 (2017).

[23] C. Macchiavello and M. F. Sacchi, Phys. Rev. Lett. 116, 140501 (2016).

[24] C. Macchiavello and M. F. Sacchi, Phys. Rev. A 94, 052333 (2016).

[25] B. W. Schumacher and M. A. Nielsen, Phys. Rev. A 54, 2629 (1996).

[26] B. W. Schumacher, Phys. Rev. A 54, 2614 (1996).

[27] See the Supplemental Material, which includes Refs. [23], 30].

[28] This can also be seen by the following identity for the expectations on the bipartite output state $\left\langle\sigma_{\alpha} \otimes \sigma_{\beta}\right\rangle=\operatorname{Tr}\left[\left(\mathcal{I}_{A} \otimes\right.\right.$ $\left.\mathcal{E})\left(\left|\Phi^{+}\right\rangle\left\langle\Phi^{+}\right|\right)\left(\sigma_{\alpha} \otimes \sigma_{\beta}\right)\right]=\frac{1}{2} \operatorname{Tr}\left[\sigma_{\beta} \mathcal{E}\left(\sigma_{\alpha}^{\tau}\right)\right]$, where $\tau$ denotes the transposition. The expectation values $\left\langle\sigma_{\alpha} \otimes \sigma_{\alpha}\right\rangle$ and therefore also the probabilities in Eq. (5) can then be obtained by considering only the system qubit, preparing it in the eigenstates of $\sigma_{\alpha}^{\tau}$ with equal probabilities, and measuring $\sigma_{\alpha}$ at the output of the channel.

[29] A. Fedrizzi, T. Herbst, A. Poppe, T. Jennewein, and A. Zeilinger, Optics Express 15, 15377 (2007).

[30] D. James, P. Kwiat, W. Munro, and A. White, Phys. Rev. A, 64, 052312 (2001).

[31] N. K. Bernardes, Á. Cuevas, A. Orieux, C. H. Monken, P. Mataloni, F. Sciarrino, and M. F. Santos, Sci. Rep. 5, 17520 (2015).

[32] M. A. Nielsen and I. L. Chuang, Quantum Computation and Quantum Information: 10th Anniversary Edition (Cambridge University Press, Cambridge, 2010).

[33] E. Desurvire, Classical and Quantum Information Theory: An Introduction for the Telecom Scientist (Cambridge University Press, Cambridge, 2009).

[34] Á. Cuevas, A. Mari, A. De Pasquale, A. Orieux, M. Massaro, F. Sciarrino, P. Mataloni, and V. Giovannetti, Phys. Rev. A, 96, 012314 (2017).

[35] M. P. Almeida, F. de Melo, M. Hor-Meyll, A. Salles, S. P. Walborn, P. H. Souto Ribeiro, and L. Davidovich, Science 316, 579 (2007).

[36] V. Giovannetti and R. Fazio, Phys.Rev. A 71, 032314 (2005).

[37] D. Di Vincenzo, P. W. Shor, and J. Smolin, Phys. Rev. A 57, 830 (1998).

[38] D. James, P. Kwiat, W. Munro, and A. White, Phys. Rev. Lett. A 64, 052312 (2001). 